\title{
The heme precursor 5-aminolevulinic acid disrupts the Warburg effect in tumor cells and induces caspase-dependent apoptosis
}

\author{
YUTA SUGIYAMA $^{1}$, YUICHIRO HAGIYA ${ }^{1}$, MOTOWO NAKAJIMA ${ }^{2}$, MASAHIRO ISHIZUKA ${ }^{2}$, \\ TOHRU TANAKA ${ }^{2}$ and SHUN-ICHIRO OGURA ${ }^{1}$ \\ ${ }^{1}$ Graduate School of Bioscience and Biotechnology, Tokyo Institute of Technology, Yokohama, \\ Kanagawa 226-8501; ${ }^{2}$ SBI Pharmaceuticals Co., Ltd., Tokyo 106-6020, Japan
}

Received August 22, 2013; Accepted October 2, 2013

DOI: 10.3892/or.2013.2945

\begin{abstract}
Our previous study demonstrated that 5-aminolevulinic acid (ALA) administered to mice stimulates oxidative phosphorylation by upregulation of the mitochondrial respiratory chain complex IV enzyme cytochrome $c$ oxidase (COX). The present study investigated whether ALA disrupts the Warburg effect, which represents a shift in ATP generation from oxidative phosphorylation to glycolysis, protecting tumor cells against oxidative stress-mediated apoptosis. The human lung carcinoma cell line A549 exposed to ALA exhibited enhanced oxidative phosphorylation, which was indicated by an increase in COX protein expression and oxygen consumption. Furthermore, ALA suppressed glycolysis-mediated acidosis. This normalization of the ATP metabolic pathways significantly increased the generation of superoxide anion radical $\left(\mathrm{O}_{2}{ }^{\circ}\right)$ and the functional expression of active caspase-3, leading to caspase-dependent apoptosis. These data demonstrate that ALA inhibits the Warburg effect and induces cancer cell death. Use of this endogenous compound might constitute a novel approach to cancer therapy.
\end{abstract}

\section{Introduction}

Tumor cells exhibit a metabolic phenotype named the Warburg effect, whereby ATP is primarily generated by glycolysis, instead of oxidative phosphorylation, even under

Correspondence to: Professor Shun-Ichiro Ogura, Department of Bioengineering, Graduate School of Bioscience and Biotechnology, Tokyo Institute of Technology, 4259 B102 Nagatsuta-cho, Yokohama, Kanagawa 226-8501, Japan

E-mail: sogura@bio.titech.ac.jp

Abbreviations: COX, cytochrome $c$ oxidase; ALA, 5-aminolevulinic acid

Key words: 5-aminolevulinic acid, Warburg effect, cytochrome $c$ oxidase, porphyrin metabolism, electron transport chain normoxic conditions (1). In normal cells, oxidative phosphorylation is carried out by a series of protein complexes embedded in the mitochondrial inner membrane. These electron transport chains transfer electrons from donors to acceptors, such as oxygen. These redox reactions generate energy used to convert ADP to ATP. Although glycolysis is a less efficient pathway for generating ATP, it allows cell growth under hypoxic conditions, encountered in expanding tumors. Furthermore, oxidative phosphorylation generates reactive oxygen species (ROS), known to cause apoptosis in tumor cells (2). Therefore, the Warburg effect further allows tumor cells to suppress ROS-mediated apoptosis. Bonnet et al (3) discovered that dichloroacetate (DCA), a drug used to treat human lactic acidosis, inhibits mitochondrial pyruvate dehydrogenase kinase and selectively kills cancer cells by normalizing cancer-associated metabolic properties. The drug suppresses glycolysis and promotes oxidative phosphorylation, thereby increasing mitochondrial ROS-mediated apoptosis in tumor cells (3-6). These data highlight the importance of suppressing oxidative phosphorylation in tumor cells.

The enzyme cytochrome $c$ oxidase (COX; complex IV) plays a key role at the end of the electron transport chain (7). This hemoprotein uses a heme group as an active site to oxidize cytochrome $c$. In most tumor cells, COX activities are suppressed. We recently reported that 5 -aminolevulinic acid (ALA) increases COX activity in mouse liver (8). This compound is widely distributed in plant and animal cells and is a common precursor of tetrapyrroles, such as the active core of hemoglobin. In animal cells, ALA is generated from glycine and succinyl CoA by the ALA synthase in the mitochondria. ALA administration induces the cancer-specific accumulation of fluorescent porphyrins (9). Therefore, fluorescence diagnosis, also named photodynamic diagnosis, is based on the different ALA-induced fluorescent signatures of normal and cancer tissues (10).

The present study tested the impact of ALA on the Warburg effect in vitro to determine whether this compound might induce apoptosis in cancer cells by restoring normal metabolic activities. The human lung carcinoma cell line A549 was exposed to ALA to determine its impact on oxidative phosphorylation, glycolysis, and caspase-dependent apoptosis. The 
present study demonstrates the potential of this endogenous compound for the development of new cancer treatments.

\section{Materials and methods}

Materials. The substrate ALA hydrochloride was purchased from Cosmo Oil Co., Ltd. (Tokyo, Japan). RPMI-1640 medium and antibiotic-antimycotic mixture (ABAM; penicillin-streptomycin-amphotericin B) were obtained from Nacalai Tesque (Kyoto, Japan). Fetal bovine serum (FBS) was purchased from Invitrogen (Carlsbad, CA, USA). All other chemicals were of analytical grade.

Cells. The human lung carcinoma cell line A549 was purchased from Riken BRC Cell Bank (Tsukuba, Ibaraki, Japan). Cells were grown in RPMI-1640 medium supplemented with $10 \% \mathrm{FBS}$ and $\mathrm{ABAM}$ at $37^{\circ} \mathrm{C}$ in an incubator with a controlled humidified atmosphere composed of $5 \% \mathrm{CO}_{2}$.

Determination of cell survival. The A549 cells ( $2 \times 10^{4}$ cells) were incubated at $37^{\circ} \mathrm{C}$ in RPMI-1640 medium containing ALA, with or without $20 \mu \mathrm{M}$ of the caspase inhibitor Z-VAD-FMK (Enzo Life Sciences, Farmingdale, NJ, USA), under $5 \% \mathrm{CO}_{2}$ for $72 \mathrm{~h}$ in the dark to avoid photocytotoxicity from the generated porphyrins. After $72 \mathrm{~h}$, the number of living cells was determined by the trypan blue dye exclusion assay.

Preparation of cell lysate samples. Confluent cultures were washed with ice-cold PBS (-) and then treated with lysis buffer A (50 mM Tris-HCl pH 7.4, 1 mM DTT, $1 \% \mathrm{v} / \mathrm{v}$ Triton X-100) containing a protease inhibitor cocktail (Nacalai Tesque). The preparation was homogenized by 10 passages through a $27-\mathrm{G}$ needle. The homogenate was centrifuged at $1,000 \times \mathrm{g}$ for $10 \mathrm{~min}$ at $4^{\circ} \mathrm{C}$, and the resulting supernatant was collected as the cell lysate. Protein concentration of the supernatant was determined by the Bradford assay (Bio-Rad Laboratories, Richmond, CA, USA) using bovine serum albumin as a standard.

SDS-PAGE and western blot analysis. SDS-PAGE and western blot analysis were performed as described previously (11), with some modifications. In brief, the cell lysate was mixed with SDS-PAGE sample buffer containing $10 \% \mathrm{v} / \mathrm{v}$ 2-mercaptoethanol (Daiichi Pure Chemicals Co., Ltd., Tokyo, Japan), and proteins were electrophoretically separated on a $15 \%$ polyacrylamide gel. The gel was electroblotted onto a polyvinylidene fluoride membrane (Millipore, Bedford, MA, USA). The membrane was incubated in blocking solution containing $5 \% \mathrm{w} / \mathrm{v}$ skim milk in TTBS $(20 \mathrm{mM}$ Tris- $\mathrm{HCl} \mathrm{pH} 7.4,150 \mathrm{mM}$ $\mathrm{NaCl}, 0.05 \% \mathrm{v} / \mathrm{v}$ Tween 20 ) at $4^{\circ} \mathrm{C}$ overnight. The specific antibodies used were: human anti-heme oxygenase-1 (HO-1) monoclonal antibody (1:200; Abcam, Cambridge, UK), human catalase monoclonal antibody, human manganese superoxide dismutase (MnSOD) polyclonal antibody, human caspase-3 monoclonal antibody and human COX IV-1 monoclonal antibody (all at 1:200 dilution; Santa Cruz Biotechnology Inc., Dallas, TX, USA). Human GAPDH monoclonal antibody (1:1,000; American Research Products Inc., Waltham, MA, USA) was used as an internal standard. The secondary antibody used was anti-mouse IgG horseradish peroxidase (HRP)- conjugated antibody (1:3,000; Cell Signaling Technology Inc., Danvers, MA, USA). HRP-dependent luminescence was developed using Western Lightning ${ }^{\mathrm{TM}}$ Chemiluminescent Reagent Plus (PerkinElmer Life and Analytical Sciences Inc., Waltham, MA, USA) and detected using the lumino-image analyzer ImageQuant LAS-4000 mini (GE Healthcare Life Sciences UK, Little Chalfont, UK). Chemiluminescence intensity was quantified using an ImageQuant ${ }^{\mathrm{TM}}$ TL Analysis Toolbox (GE Healthcare Life Sciences).

Measurements of dissolved oxygen concentrations in cultured medium. Dissolved oxygen concentrations in cultured medium were determined using a 24-channel SDR SensorDish ${ }^{\circledR}$ Reader (PreSens Precision Sensing GmbH, Regensburg, Germany), with some modifications (12). In brief, A549 cells were seeded on a 24-well OxoDish ${ }^{\circledR}\left(5 \times 10^{4}\right.$ cells/well; PreSens Precision Sensing $\mathrm{GmbH}$ ) and cultured at $37^{\circ} \mathrm{C}$ under $5 \% \mathrm{CO}_{2}$ for $16 \mathrm{~h}$. Next, the incubation medium was replaced with $1,000 \mu \mathrm{l}$ of fresh medium covered with $800 \mu \mathrm{l}$ of liquid paraffin as a gas barrier. The cells were cultured at $37^{\circ} \mathrm{C}$ under atmosphere, and measurements of dissolved oxygen concentrations were performed for $24 \mathrm{~h}$ at $1-\mathrm{h}$ intervals.

Measurements of $\mathrm{pH}$ of the cultured medium. Since glycolysis causes lactic acidosis in vivo and a decrease in $\mathrm{pH}$ of culture medium in vitro, the impact of ALA on the glycolytic pathway was measured by monitoring culture medium $\mathrm{pH}$ using the 24-channel SDR SensorDish Reader (12). In brief, A549 cells were seeded on a 24 -well HydroDish ${ }^{\circledR}\left(5 \times 10^{4}\right.$ cells/well, PreSens Precision Sensing $\mathrm{GmbH}$ ) and cultured at $37^{\circ} \mathrm{C}$ under $5 \% \mathrm{CO}_{2}$ for $16 \mathrm{~h}$. Next, the incubation medium was replaced with $800 \mu \mathrm{l}$ of fresh buffer-free RPMI-1640 medium without $\mathrm{NaHCO}_{3}$, covered with $800 \mu \mathrm{l}$ of liquid paraffin as a gas barrier. The cells were cultured at $37^{\circ} \mathrm{C}$ under atmosphere, and $\mathrm{pH}$ measurements were performed for $24 \mathrm{~h}$ at $1-\mathrm{h}$ intervals.

Detection of superoxide anion radicals. Intracellular superoxide anion radical $\left(\mathrm{O}_{2}^{-*}\right)$ levels were detected using a Diogenes Cellular Luminescence Enhancement System (National Diagnostics, Somerville, NJ, USA) as described previously (13), with some modifications. The Diogenes kit is a supersensitive version of the traditional luminol assay that measures oxidation levels directly proportional to chemiluminescence. Cells seeded on a 96 -well plate $\left(3 \times 10^{4}\right.$ cells/well) were cultured overnight at $37^{\circ} \mathrm{C}$ under $5 \% \mathrm{CO}_{2}$. Next, the incubation medium was replaced with fresh medium containing ALA. The cells were further incubated in the dark for 2 or $4 \mathrm{~h}$, and the medium was replaced with $100 \mu \mathrm{l}$ of Diogenes reagent. Following incubation at $37^{\circ} \mathrm{C}$ for $10 \mathrm{~min}$, the luminescence level of the reagent was measured using a luminescence microplate reader (LMax II; Molecular Devices, Sunnyvale, CA, USA).

Measurements of caspase-3 activity. The enzymatic assay was performed using a Caspase-3 Colorimetric Assay kit (Medical and Biological Laboratories Co., Ltd., Nagoya, Japan) as described previously (14), with some modifications. In brief, A549 cells $\left(2 \times 10^{6}\right.$ cells) were lysed with $250 \mathrm{ml}$ of lysis buffer on ice for $10 \mathrm{~min}$. Following centrifugation $(10,000 \mathrm{x} \mathrm{g}, 10 \mathrm{~min}$, $4^{\circ} \mathrm{C}$ ), the supernatant was collected for the caspase-3 assay. A total of $100 \mu \mathrm{g}$ of protein was diluted in $50 \mu \mathrm{l}$ of cell lysis 


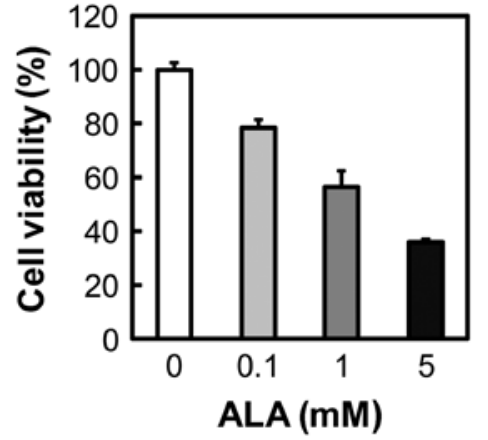

Figure 1. Cell survival of A549 cells following incubation with 5-aminolevulinic acid (ALA). A549 cells were incubated with ALA for $72 \mathrm{~h}$ and cell survival was determined by the trypan blue dye exclusion assay.
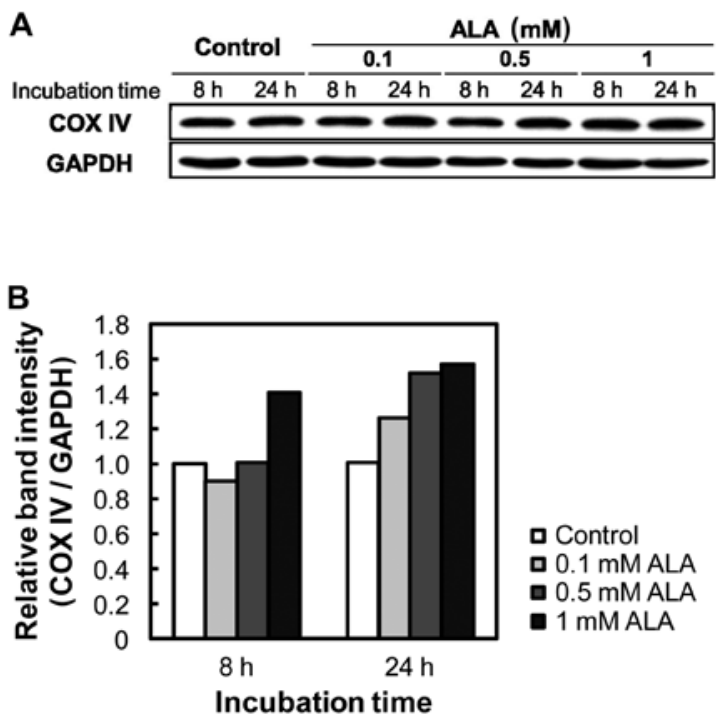

Figure 2. Western blot analysis of A549 cells following incubation with 5-aminolevulinic acid (ALA). Subunit IV of COX was detected by western blot analysis (A) and the intensity of bands was quantified (B).

buffer and mixed with $50 \mu \mathrm{l}$ of $2 \mathrm{X}$ reaction buffer (containing $10 \mathrm{mM}$ DTT) and $5 \mu \mathrm{l}$ of substrate (10 mM Asp-Glu-Val-Aspp-nitroanilide; DEVD-pNA). Following incubation at $37^{\circ} \mathrm{C}$ for $24 \mathrm{~h}$, absorbance was read at $405 \mathrm{~nm}$ using the microtiter plate reader Multiskan ${ }^{\circledR}$ FC (Thermo Fisher Scientific, San Jose, CA, USA).

\section{Results}

Impact of ALA on the survival of A549 cells. The impact of ALA on the survival of cancer cells was tested using A549 cells. A significant decrease in cell survival was detected following a 72-h exposure to ALA (Fig. 1). Since all experiments were performed in complete darkness, cell death was not caused by photocytotoxicity of the porphyrins generated from ALA. By contrast, the caspase inhibitor Z-VAD-FMK prevented cell death, thereby supporting a mechanism of ALA-mediated apoptosis in cancer cells.

Impact of ALA on the Warburg effect in A549 cells. To investigate the effect of ALA on the Warburg effect, key elements
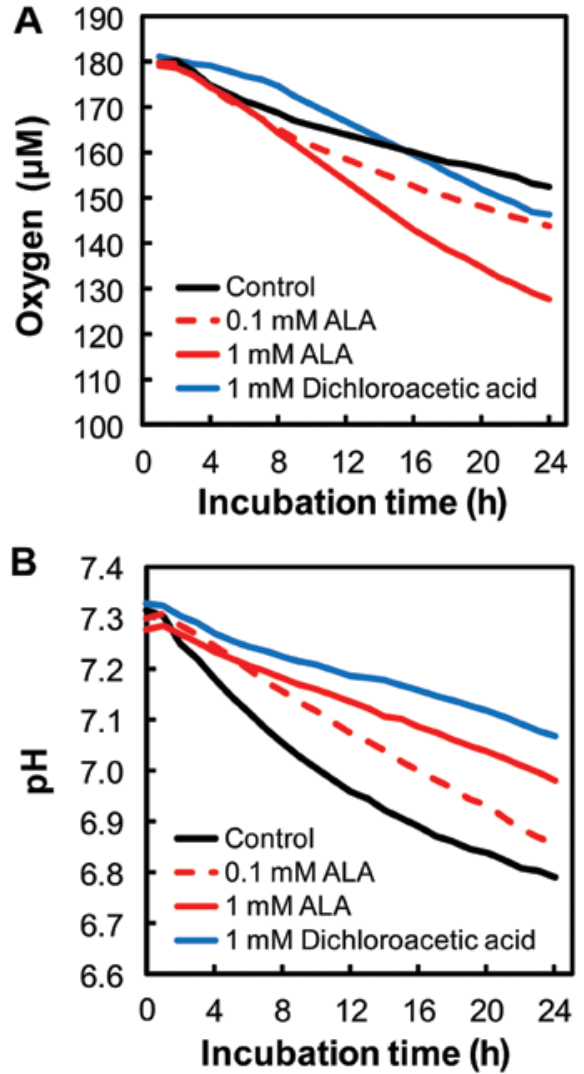

Figure 3. Metabolic change in A549 cells following incubation with 5-aminolevulinic acid (ALA). Aerobic respiration was evaluated by measuring oxygen consumption (A) and glycolysis was evaluated by measuring the $\mathrm{pH}$ of cultured medium (B).

of oxidative phosphorylation and glycolysis were monitored in A549 cells pre- and post-exposure to ALA. First, ALA induced an increase in COX protein expression (Fig. 2), supporting a stimulating effect on oxidative phosphorylation. This result is consistent with the result from our previous study, where COX expression in mouse liver was increased following ALA administration (8). In addition, oxygen consumption was significantly higher following ALA exposure (Fig. 3A). Furthermore, the oxygen consumption ratio induced by ALA was higher than the ratio induced by DCA, which is a common enhancer of oxidative phosphorylation. Taken together, these data suggest that the increase in the oxygen consumption level measured in A549 cells following ALA exposure requires the induction of key proteins supporting oxidative phosphorylation, including COX.

Since glycolysis causes acidosis, the impact of ALA on this pathway was determined by monitoring the $\mathrm{pH}$ of the A549 cell culture medium during a 24-h incubation period (Fig. 3B). In control cultures, the rate of $\mathrm{pH}$ decay was typical of cancer cells. The presence of ALA prevented any significant decrease in $\mathrm{pH}$ during the 24-h period, suggesting that the compound suppressed glycolysis in A549 cancer cells.

Impact of ALA on ROS generation. The impact of ALA on oxidative phosphorylation was additionally tested in A549 cells with respect to the generation of ROS, namely $\mathrm{O}_{2}{ }^{\circ-}$. Fig. 4 shows that ALA significantly increased $\mathrm{O}_{2}{ }^{-}$-generation over $4 \mathrm{~h}$. It is well known that ROS generation stimulates the 


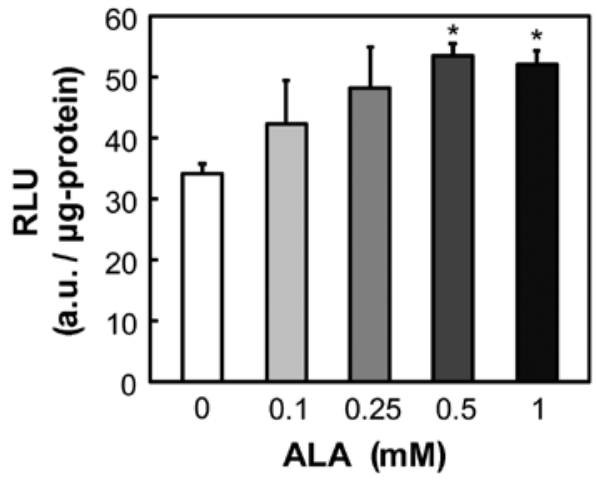

Figure 4. Generation of superoxide anion radical $\left(\mathrm{O}_{2}^{*}\right)$ from A549 cells following incubation with 5-aminolevulinic acid (ALA). $\mathrm{O}_{2}{ }^{--}$levels were measured using a Diogenes Cellular Luminescence Enhancement System. Cells were incubated with or without ALA for $4 \mathrm{~h}$. ${ }^{*} \mathrm{p}<0.05$, statistical significance of differences between paired groups.

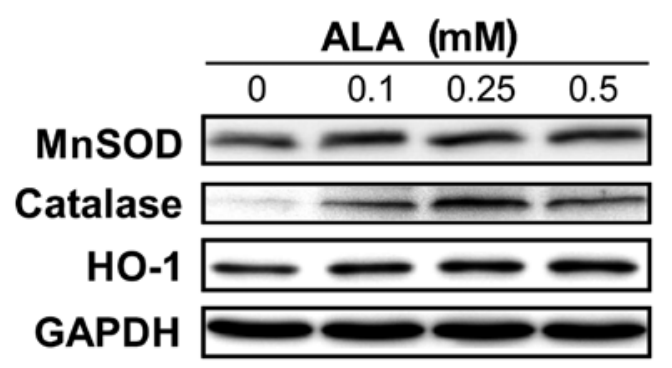

Figure 5. Expression of scavenger enzymes. Scavenger enzymes manganese superoxide dismutase (MnSOD), catalase and heme oxygenase-1 (HO-1) were detected in A549 cells by western blot analysis following a 24-h incubation with 5-aminolevulinic acid (ALA).

expression of free radical scavenging enzymes. Western blot analysis (Fig. 5) shows that ALA stimulates MnSOD, catalase and HO-1 protein expression. Altogether, these data show that ALA-mediated ROS generation was sufficient to recruit additional scavenger enzymes to protect cancer cells against oxidative stress-mediated apoptosis. Therefore, ALA significantly stimulated the oxidative phosphorylation pathway in A549 cancer cells.

Induction of apoptosis by ALA. Since ROS generation induces apoptosis in cancer cells (15), experiments were performed to test whether ALA induces apoptosis in A549 cells. Activated caspase- 3 is a key player in the initiation of apoptosis. Fig. 6A shows that ALA induced an increase in the protein expression of activated (cleaved) caspase-3. The functional overexpression of active caspase- 3 was demonstrated by enhanced cleavage of DEVD-pNA (Fig. 6B) in the presence of ALA. Therefore, ALA increases the caspase-3 activity level in cancer cells. Furthermore, the inhibitor of caspase-dependent apoptosis, Z-VAD-FMK, prevented ALA-mediated cell death (Fig. 7). Altogether, these data demonstrate that ALA induced caspasedependent apoptosis in A549 cells.

\section{Discussion}

At present, the most important goal of cancer research teams is to discover tumor cell-specific treatments to reduce the
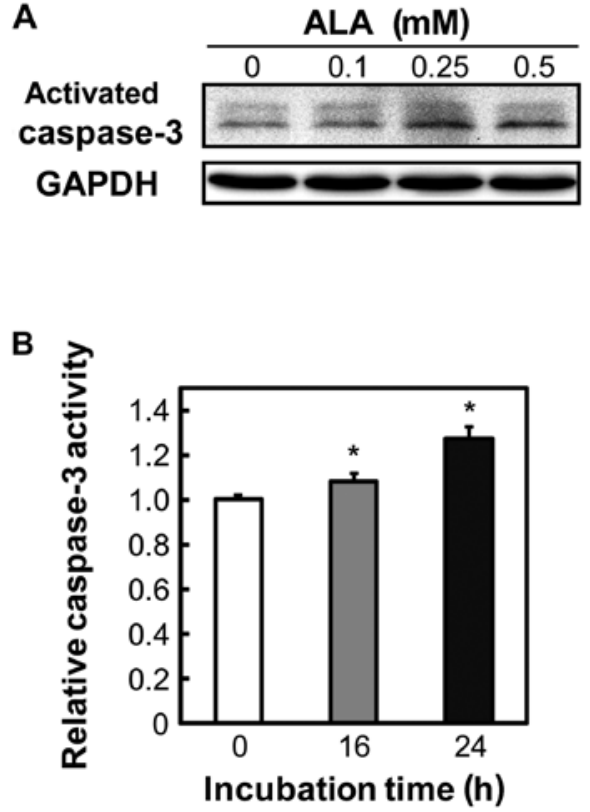

Figure 6. Measurement of activation of caspase-3. Activated caspase-3 was detected in A549 cells by western blot analysis following a 24-h incubation with 5-aminolevulinic acid (ALA) (A) and caspase-3 activity was measured using the Caspase-3 Colorimetric Assay kit following incubation with $1 \mathrm{mM}$ ALA (B). ${ }^{*} \mathrm{p}<0.05$, statistical significance of differences between paired groups.

serious side-effects of chemotherapy. Recent developments in molecular biology and metabolomics have shown that tumor cells exhibit a unique metabolic phenotype, named the Warburg effect, which represents a shift in ATP generation from oxidative phosphorylation to glycolysis (16). For tumor cells, it is important to suppress oxidative phosphorylation since this pathway generates ROS, causing apoptosis and cell death (2). The importance of this phenomenon is illustrated by the effect of DCA, which selectively kills cancer cells by normalizing cancer-associated metabolic properties and stimulating ROS-mediated apoptosis (3-6).

The present study showed that the endogenous compound ALA exhibits properties similar to DCA in cancer cells, i.e., restoration of oxidative phosphorylation, suppression of glycolysis, and ROS-mediated apoptosis. To our knowledge, this is the first in vitro study showing that ALA causes tumor apoptosis via enhancement of oxidative phosphorylation. In a previous study, we showed that in mouse liver, ALA increases the expression and activity of COX, which is an indicator of oxidative phosphorylation (8). The present study goes further by showing that ALA affects both aspects of the Warburg effect by increasing oxidative phosphorylation and suppressing glycolysis. Therefore, ALA stimulates ROS-mediated apoptosis in tumor cells and reduces their capacity to grow in the low oxygen (hypoxic) environment of the tumor mass. These data strongly support the potential of ALA as a selective antitumor agent.

ALA is widely used in tumor therapy (photodynamic therapy) and diagnosis (photodynamic diagnosis) since tumor cell-specific fluorescent porphyrin accumulates following ALA administration (9). However, ALA has not yet been considered as a therapeutic drug. The main effect of porphyrin accumulation is the overexpression of the peptide transporter 


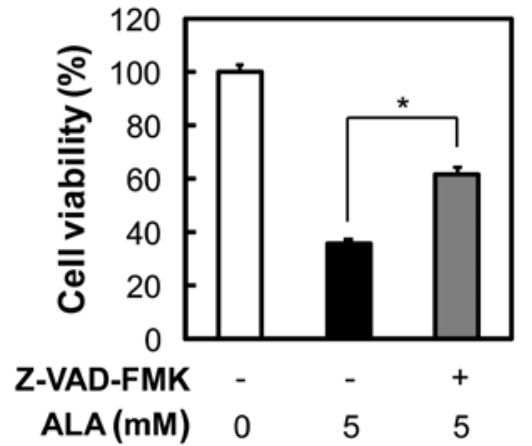

Figure 7. The effect of caspase inhibitor on 5-aminolevulinic acid (ALA)-induced cell death. A549 cells were incubated with or without ALA and Z-VAD-FMK for $72 \mathrm{~h}$ and cell survival was determined by the trypan blue dye exclusion assay.

PEPT1 or PEPT2 (ALA influx transporter) in tumor cells and tissues $(17,18)$. Therefore, the cytotoxic effects of ALA would only be evident in tumor cells. Moreover, no side-effect of ALA has been reported in normal cells (9). These findings support ALA as a high-potential, tumor-specific antitumor agent. Further studies are needed to examine the activity of ALA in vivo using experimental xenograft models and clinical tumor specimens.

\section{Acknowledgments}

We thank Professors Eiry Kobatake and Masayasu Mie for measurements of superoxide anion radicals.

\section{References}

1. Warburg O: On the origin of cancer cells. Science 123: 309-314, 1956.

2. Pan JG and Mak TW: Metabolic targeting as an anticancer strategy: dawn of a new era? Sci STKE 381: pe14, 2007.

3. Bonnet S, Archer SL, Allalunis-Turner J, Haromy A, Beaulieu C, Thompson R, Lee CT, Lopaschuk GD, Puttagunta L, Bonnet $\mathrm{S}$, et al: A mitochondria- $\mathrm{K}^{+}$channel axis is suppressed in cancer and its normalization promotes apoptosis and inhibits cancer growth. Cancer Cell 11: 37-51, 2007.

4. Michelakis ED, Webster L and Mackey JR: Dichloroacetate (DCA) as a potential metabolic-targeting therapy for cancer. $\mathrm{Br} \mathbf{J}$ Cancer 99: 989-994, 2008.

5. Sutendra G, Dromparis P, Kinnaird A, Stenson TH, Haromy A, Parker JM, et al: Mitochondrial activation by inhibition of PDKII suppresses HIF1a signaling and angiogenesis in cancer. Oncogene 32: 1638-1650, 2012.
6. Papandreou I, Goliasova T and Denko NC: Anticancer drugs that target metabolism: Is dichloroacetate the new paradigm? Int $\mathrm{J}$ Cancer 128: 1001-1008, 2011.

7. Fontanesi F, Soto IC, Horn D and Barrientos A: Assembly of mitochondrial cytochrome c-oxidase, a complicated and highly regulated cellular process. Am J Physiol Cell Physiol 291: 1129-1147, 2006.

8. Ogura S, Maruyama K, Hagiya Y, Sugiyama Y, Tsuchiya K, Takahashi K, Abe F, Tabata K, Okura I, Nakajima M and Tanaka T: The effect of 5-aminolevulinic acid on cytochrome c oxidase activity in mouse liver. BMC Res Notes 4: 66, 2011.

9. Ishizuka M, Abe F, Sano Y, Takahashi K, Inoue K, Nakajima M, Kohda T, Komatsu N, Ogura S and Tanaka T: Novel development of 5-aminolevurinic acid (ALA) in cancer diagnoses and therapy. Int Immunopharmacol 11: 358-365, 2011.

10. Stummer W, Pichlmeier U, Meinel T, Wiestler OD, Zanella F, Reulen HJ; ALA-Glioma Study Group: Fluorescence-guided surgery with 5-aminolevulinic acid for resection of malignant glioma: a randomised controlled multicentre phase III trial. Lancet Oncol 7: 392-401, 2006.

11. Ogura S, Kaneko K, Miyajima S, Ohshima K, Yamaguchi K and Mochizuki T: Proneurotensin/neuromedin N secreted from small cell lung carcinoma cell lines as a potential tumor marker. Proteomics Clin Appl 2: 1620-1627, 2008.

12. Naciri M, Kuystermans D and Al-Rubeai M: Monitoring pH and dissolved oxygen in mammalian cell culture using optical sensors. Cytotechnology 57: 245-250, 2008.

13. Adachi R, Takeuchi K and Suzuki K. Antisense oligonucleotide to cofilin enhances respiratory burst and phagocytosis in opsonized zymosan-stimulated mouse macrophage J774.1 cells. J Biol Chem 277: 45566-45571, 2002.

14. Li CY, Lee JS, Ko YG, Kim JI and Seo JS: Heat shock protein 70 inhibits apoptosis downstream of cytochrome c release and upstream of caspase-3 activation. J Biol Chem 275: 25665-25671, 2000.

15. Wang $X$ : The expanding role of mitochondria in apoptosis Genes Dev 15: 2922-2933, 2001.

16. Hirayama A, Kami K, Sugimoto M, Sugawara M, Toki N, Onozuka H, Kinoshita T, Saito N, Ochiai A, Tomita M, Esumi H and Soga T: Quantitative metabolome profiling of colon and stomach cancer microenvironment by capillary electrophoresis time-of-flight mass spectrometry. Cancer Res 69: 4918-4925, 2009.

17. Hagiya Y, Endo Y, Yonemura Y, Takahashi K, Ishizuka M, Abe F, Tanaka T, Okura I, Nakajima M, Ishikawa T and Ogura S: Pivotal roles of peptide transporter PEPT1 and ATP-binding cassette $(\mathrm{ABC})$ transporter ABCG2 in 5-aminolevulinic acid (ALA)-based photocytotoxicity of gastric cancer cells in vitro. Photodiagnosis Photodyn Ther 9: 204-214, 2012.

18. Hagiya Y, Fukuhara H, Matsumoto K, Endo Y, Nakajima M, Tanaka T, Okura I, Kurabayashi A, Furihata M, Inoue K, Shuin T and Ogura S: Expression levels of PEPT1 and ABCG2 play key roles in 5-aminolevulinic acid (ALA)-induced tumor-specific protoporphyrin IX (PpIX) accumulation in bladder cancer. Photodiagnosis Photodyn Ther 10: 288-295, 2013. 